\begin{tabular}{|c|c|c|}
\hline \multirow{3}{*}{$\begin{array}{r}\text { Case Reports in } \\
\text { Gastroenterology }\end{array}$} & \multirow{2}{*}{\multicolumn{2}{|c|}{ Case Rep Gastroenterol 2017;11:624-632 }} \\
\hline & & \\
\hline & $\begin{array}{l}\text { DOI: 10.1159/000481302 } \\
\text { Published online: October 30, } 2017\end{array}$ & $\begin{array}{l}\text { (c) } 2017 \text { The Author(s) } \\
\text { Published by S. Karger AG, Basel } \\
\text { www.karger.com/crg }\end{array}$ \\
\hline & \multicolumn{2}{|c|}{$\begin{array}{l}\text { This article is licensed under the Creative Commons Attribution-NonCommercial } 4.0 \\
\text { International License (CC BY-NC) (http://www.karger.com/Services/OpenAccessLicense). } \\
\text { Usage and distribution for commercial purposes requires written permission. }\end{array}$} \\
\hline
\end{tabular}

\title{
A Silent Asymptomatic Solid Pancreas Tumor in a Nonsmoking Athletic Female: Pancreatic Ductal Adenocarcinoma
}

\author{
Kyawzaw Lin ${ }^{a}$ Aung Naing Lin ${ }^{a}$ Sithu Lin ${ }^{a}$ Thinzar Lin ${ }^{a}$ \\ Ying Xian Liu ${ }^{b}$ Madhavi Reddy ${ }^{c}$ \\ anternal Medicine Department, The Brooklyn Hospital Center, Affiliate of the Mount Sinai \\ Hospital, Brooklyn, NY, USA; 'bathological Department, The Brooklyn Hospital Center, \\ Affiliate of the Mount Sinai Hospital, Brooklyn, NY, USA; ${ }^{C}$ GI Department, The Brooklyn \\ Hospital Center, Affiliate of the Mount Sinai Hospital, Brooklyn, NY, USA
}

\section{Keywords}

Solid pancreatic tumor · Hepatobiliary tumor · Gastroenterology · Pancreatic ductal adenocarcinoma

\begin{abstract}
A silent solid endocrine tumor of pancreas, intraductal adenocarcinoma of pancreas, is the fourth leading cancer-related death in the US. However, it is expected to become the third leading cause by 2030 owing to delayed diagnosis and slow progress in management. Chronic pancreatitis is at risk for pancreatic ductal adenocarcinoma (PDAC). PDAC is diagnostic with transabdominal sonogram, blood test such as carbohydrate antigen 19-9 (CA 19-9), and imaging. PDAC has a dismal prognosis. The survival rate in 5 years is barely $6 \%$, while late detection rate is $80-85 \%$ with unresectable stage upon diagnosis. Here, we present a 51 year-old asymptomatic female with intermittent constipation and abdominal pain for 1 month with obstructive jaundice with PDAC with liver metastasis.
\end{abstract}




\section{Case Reports in Gastroenterology}

Case Rep Gastroenterol 2017;11:624-632 DOI: $10.1159 / 000481302$

(c) 2017 The Author(s). Published by S. Karger AG, Basel www.karger.com/crg

Lin et al.: A Silent Asymptomatic Solid Pancreas Tumor in a Nonsmoking Athletic Female: Pancreatic Ductal Adenocarcinoma

\section{Introduction}

Pancreatic ductal adenocarcinoma (PDAC) is the commonly diagnosed exocrine tumor of pancreas (nearly 85\%). The highest incidence is seen in Maoris, native Hawaiians, and African-American populations. It is the eighth leading cause of mortality from cancer in male and female. The incidence is relatively rare before 45 , but sharply rises after 45 . The incidence is trending up in the setting of high prevalence in obesity, elderly population, and other metabolic related syndrome. Non-blood 0 groups (blood types $\mathrm{A}, \mathrm{B}$, and $\mathrm{AB}$ ) are at high risk and the association between those groups and PDAC is presumptively related to $\mathrm{H}$. pylori colonization in the hepatobiliary system [1]. Metabolic related syndrome such as diabetes mellitus (DM) and obesity predisposes to PDAC in the setting of low plasma adiponectin level. However, new onset of DM can be due to underlying asymptomatic PDAC [2].

More than $90 \%$ of all pancreatic tumors are ductal adenocarcinomas and its variants. Presenting symptoms are vague such as bloating, indigestion, weight loss, pain, jaundice, and sometimes pancreatitis. However, there are no early alarming signs of pancreatic cancer. New onset of DM might alert early stage of pancreatic cancer, especially in the elderly population.

Pancreatic protocol CT and MRI are the preferred imaging tool for determination of resectability. In case of suspected tumor invisible on CT scan or in determined hepatic lesions on CT scan, MRI with contrast can be used for staging purpose. Multiphasic pancreatic CT scan can be useful to detect vascular invasion by tumors or visualization of structures such as mesenteric artery, celiac axis, or mesenteric veins that can predict the resectability. ERCP is mainly therapeutic and used for palliative biliary obstruction and for stent placement.

Ultrasound (US) cannot be used as routine tool for staging purposes or to assess vascular involvement as it has operator dependence. Pathological confirmation is done not for surgery but prior to administration of adjuvant chemotherapy for locally unresectable advanced tumors or metastatic tumor. However, endoscopic ultrasound-guided fine needle aspiration (EUS-FNA) is preferred over CT-guided FNA for resectable tumors. If tissue biopsy does not yield any satisfactory report, another attempt should be considered. A confirmed diagnosis is a must before chemotherapy. Carbohydrate antigen 19-9 (CA 19-9) is used as a diagnostic maker in the setting of specificity $80-90 \%$ in symptomatic patients, while sensitivity is $79-81 \%[3]$.

Gemcitabine monotherapy is used for patients with good performance status with locally advanced tumor. Chemotherapy with systemic chemotherapy or stereotactic body radiation therapy is used for unresectable stages with good performance status. Partial or total pancreatectomy is the unique curative option for PDAC but only one-fifth of the cases are candidates due to late presentation or late diagnosis. Survival rate after surgery is only 25$30 \%$ if node negative while for node-positive cases, it is $10 \%$ in patients with resectable stages. For resectable pancreatic tail tumor, distal pancreatectomy should be attempted, while total pancreatectomy can be done for tumor diffusely involving the whole pancreas or at multiple sites in the pancreas. Whipple's procedure (pancreatoduodenectomy) is reserved for pancreas head tumor.

Strongest prognostic factors of 10-year survival include (1) negative marginal status, (2) small tumor size, and (3) negative lymph node metastasis [4]. The current recommendations for status post resectable pancreas tumor include assessment of symptoms, CA 19-9, and CT scan with contrast every 3-6 months after surgical resection for 2 years. 


\section{Case Report}

A 51-year-old female in healthy athletic state presented to the emergency department with constipation and abdominal pain on and off for 1 month duration. Abdominal pain was located in the lower abdomen, intermittent, colicky in nature, 8/10 in intensity and nonradiating, was aggravated by eating, and relieved partially by Motrin. The patient reported abdominal pain was preceded by several weeks of constipation. Later, it was associated with pruritus for 1 week, and yellowish discoloration of skin and sclera for 2 days, and deep color urine and 7-lb unintentional weight loss over a month along with decreased appetite. She denied fever, chills, Chest pain, headache, dizziness, cough, lymphadenopathy, or urinary symptoms. Her past medical history was significant with mild intermittent asthma. She denied history of smoking and alcohol or illicit drug use, but there was breast cancer in 2 paternal aunts and lung cancer in her brother. On general exam, she was a thin lady with jaundice with some scratch marks on the body, but no Murphy's sign, Gray Turner's sign, or Mayo-Robson point tenderness was elicited. CBC was within normal limit. CMP showed total bilirubin 9, alkaline phosphatase (ALP) 306, aspartate aminotransferase (AST) 246, alanine aminotransferase (ALT) 656, lipase 61, beta-HCG 3, lactic acid (LA) 0.9. Hepatitis A, B, and C was nonreactive and she was immune to hepatitis A but nonimmune to hepatitis B. PTT was 23.5, PT 12.2, INR 1.1, CA 19-9 5,329, and carcinoembryonic antigen (CEA) 4.7.

CT of the abdomen and pelvis with contrast (Fig. 1, Fig. 2) showed a 2.8-cm hypodense lesion in the hepatic dome with peripheral nodular enhancement, faint hypodense lesions, measuring up to $1.5 \mathrm{~cm}$ in the lateral segment, and mild intrahepatic biliary dilatation. Dilatation of the common duct was $0.9 \mathrm{~cm}$ with intact gallbladder and no gallstone. There was a heterogeneous, irregular solid mass involving the pancreatic body with dominant component measuring up to $8 \times 4.6 \mathrm{~cm}$ with soft tissue extension to the celiac axis and superior mesenteric artery, effacement of the splenic vein, $1.6 \mathrm{~cm}$ suspected cystic focus in the right adnexa, and a small amount of ascites.

Cholangiogram (Fig. 3) showed a long segment (3-4 cm) distal to the common bile duct (CBD) stricture with dilatation of mid and proximal CBD with dilated intrahepatic ducts. Multiple brushings were made at the stricture and sent for pathology. A $7 \mathrm{Fr} \times 9 \mathrm{~cm}$ plastic CBD stent was placed successfully transversing the stricture. ERCP (Fig. 4) report showed CBD was cannulated at the ampulla using guidewire technique. EGD report showed normal esophagus, GE junction, duodenal bulb, and second portion of the duodenum. Stent noticed in the CBD and biopsy report (Fig. 5) showed benign gastric mucosa with mild chronic gastritis. EUS report showed irregular hypoechoic mass with cystic component arising from the body of the pancreas measuring $72 \times 40.1 \mathrm{~mm}$. The mass was invaded by the celiac artery and superior mesenteric artery. Multiple small round peripancreatic lymph nodes were seen. The pancreatic mass ( $6 \mathrm{~cm}$ mass) at the body of the pancreas was examined by EUSguided fine needle aspiration biopsy (Fig. 6) and it showed malignant cells: ductal adenocarcinoma. Due to biliary obstruction pattern on liver function tests, ERCP was done and a stent was placed in the biliary tract with few brushings taken from the stricture in the biliary tract. Later, direct bilirubin was trending down from 9 to 5.2. Hepatobiliary surgery was consulted but recommended no acute intervention as the disease process was advanced and inoperable. Distal common bile duct brushing showed biliary ductal epithelium with mild reactive atypia. 


\section{Discussion}

In the US, pancreatic cancer is the fourth leading mortality from cancers in both populations. Annually, nearly 55,000 patients are diagnosed, while nearly $85 \%$ of those patients are PDAC. However, it has an unfavorable prognosis and the presentation depends on the tumor location. The common presenting signs are vague: weight loss, jaundice, and abdominal pain or even constipation. $5-10 \%$ of the patients have a segregated family history [5].

One of the recognized predisposing factors for PDAC is smoking and it is associated with $25 \%$ of the case reports. In several trials, relative risk (RR) was 1.5, was proportionately related with the number of cigarettes, and was surprisingly high in chronic heavy smokers due to homozygous deletions of glutathione S-transferase (GSST-1), the enzymes involved in metabolizing carcinogens [6, 7]. The risk declined after smoking cessation. In a prospective study, RR was 2.5 for current smokers, but the risk decreased by $50 \%$ in 2 years and the risk is the same with nonsmokers after 10-15 years of cessation. So, we can presume that cessation of smoking alone can reduce $25 \%$ of death related to pancreatic cancers in the US alone [8].

Several studies over the last 2 decades also report that long-standing DM is another risk for PDAC, although new onset of DM is observed as early presenting symptom of PDAC. Upon diagnosis, $80 \%$ of PDAC cases have glucose intolerance or DM. RR for PDAC between diabetic patients and standardized individuals is 2 [9]. Before PDAC was detected, RR risk to become diabetic in the first year is 5.38. However, the risk is inversely proportionate to the duration of DM [10]. However, those patients have better glucose control after tumor resection.

Two proposed theories are (1) adrenomedullin secreted by malignant cells cause $\beta$ cell islet dysfunction, and (2) 2030-BM peptide, a PDAC-related diabetogenic factor found in the blood of pancreatic cancer patients, causes impaired glycogen synthase activity $[11,12]$. The incidence of PDAC is reported to be low in diabetics on metformin in comparison with those on insulin [13].

Some cases are related to inhibition of tumor suppressors CDKN2A, TP53, and MADH4 or activation of K-Ras proto-oncogenes. Chronic pancreatitis is another recognized risk for PDAC and prevalence is 10-fold higher in chronic pancreatitis [14].

The commonly used serum biomarker for pancreatic cancer is the carbohydrate antigen 19-9 (CA 19-9), a Lewis antigen on MUC-1 protein class. However, CA 19-9 might be high in chronic pancreatitis, cholangitis, cirrhosis of liver, and other GI pathology. The cut-off value for pancreatic cancer is $37 \mathrm{U} / \mathrm{mL}$ in a certified clinical laboratory. In the study by Mayerle et al. [14], biomarker signature showed sensitivity $89.9 \%$, specificity $91.3 \%$, PPV 17\%, and NPV $99.8 \%$ with accuracy of $90.6 \%$ in all stages of tumors. Those biomarker signatures detected only $9 \%$ false positives compared to $23 \%$ in CA 19-9. However, Schultz et al. [15] recommend that microRNA (miRNA) panel can be used to differentiate PDAC and chronic pancreatitis. In the study, the cut-off value for CA 19-9 was $37 \mathrm{U} / \mathrm{mL}$, while for metabolomics or biomarker it is 0.384 .

CT scan or MRI can differentiate pancreatic cancer from other entities. However, ERCP or EUS is required for tissue biopsy to confirm the definite diagnosis. Complete resection is the definite treatment, though the disease process is usually late or in advanced stages upon diagnosis and the resection is impossible.

Confirmation of the tissue diagnosis using EUS-guided FNA is needed for metastatic late stages for palliative therapy or those who are not good surgical candidates. In operable adenocarcinoma, surgery can be done without any tissue confirmation. In non-obstructive or 
non-jaundice patients, without vascular or peri-neural invasion, pre-operative laparotomy is advised to rule out small metastasis that usually overlooked by imagines. For biliary obstruction without vascular invasion or perineural invasion, open laparotomy is mandatory. However, if CA 19-9 is more than 1,000 U/mL, occult distant metastasis is suggestive in CT scan or MRI, or the patients can be treated with potential palliative therapy, staging laparotomy is a must.

In conclusion, overall prognosis and outcome is poor as PDAC is usually silent and asymptomatic and presenting clinical features are very vague as in our patient. Upon accidental diagnosis or findings on imaging, the disease process is already advanced and late or inoperable. In our patient, as the tumor is located on the body of pancreas, the presenting symptoms are related to obstruction to the hepatobiliary system. The solid tumor of pancreas should always be in consideration whenever the patient presents with painless obstructive jaundice, there is new onset of DM in elderly cachectic patients, the patient is a chronic smoker with back pain, or there is recurrent chronic pancreatitis in a chronic alcoholic, and proper workup should be performed at least to rule out this silent killer of middle-age populations.

\section{Statement of Ethics}

Patient consent was obtained.

\section{Disclosure Statement}

No competing interests are declared.

\section{Author Contributions}

Dr. Kyawzaw Lin contributed equally in gathering information and writing the introduction, case report, and discussion. Dr. Aung Naing Lin, Dr. Sithu Lin, Dr. Thinzar Lin, and Dr. Reddy contributed in editing. Dr. Ying Xian Liu provided the detailed pathological reports of the case.

\section{References}

1 Risch HA, Yu H, Lu L, Kidd MS: ABO blood group, Helicobacter pylori seropositivity, and risk of pancreatic cancer: a case-control study. J Natl Cancer Inst 2010;102:502.

2 Bao Y, Giovannucci EL, Kraft P, et al: A prospective study of plasma adiponectin and pancreatic cancer risk in five US cohorts. J Natl Cancer Inst 2013;105:95

-3 Huang Z, Liu F: Diagnostic value of serum carbohydrate antigen 19-9 in pancreatic cancer: a metaanalysis. Tumor Biol 2014;35:7459-7465.

-4 Howard TJ, Krug JE, Yu J, Zyromski NJ, Schmidt CM, Jacobson LE, Madura JA, Wiebke EA, Lillemoe KD: A margin-negative R0 resection accomplished with minimal postoperative complications is the surgeon's contribution to long-term survival in pancreatic cancer. J Gastrointest Surg 2006;10:1338-1346.

Klein AP, Hruban RH, Brune KA, et al: Familial pancreatic cancer. Cancer J 2001;7:266.

Lowenfels AB, Maisonneuve P, Whitcomb DC: Risk factors for cancer in hereditary pancreatitis.

International Hereditary Pancreatitis Study Group. Med Clin North Am 2000;84:565. 
Lin et al.: A Silent Asymptomatic Solid Pancreas Tumor in a Nonsmoking Athletic Female: Pancreatic Ductal Adenocarcinoma

7 Duell EJ, Holly EA, Bracci PM, et al: A population-based, case-control study of polymorphisms in carcinogen-metabolizing genes, smoking, and pancreatic adenocarcinoma risk. J Natl Cancer Inst 2002;94:297.

8 Fuchs CS, Colditz GA, Stampfer MJ, et al: A prospective study of cigarette smoking and the risk of pancreatic cancer. Arch Intern Med 1996;156:2255.

-9 Wang F, Herrington M, Larsson J, Permert J: The relationship between diabetes and pancreatic cancer. Mol Cancer 2003;2:4.

10 Ben Q, Xu M, Ning X: Diabetes mellitus and risk of pancreatic cancer: a meta-analysis of cohort studies. Eur J Cancer 2011;47:1928-1937.

11 Aggarwal G, Ramachandran V, Javeed N: Adrenomedullin is up-regulated in patients with pancreatic cancer and causes insulin resistance in beta cells and mice. Gastroenterology. 2012;143:1510-1517.e1.

12 Basso D, Valerio A, Seraglia R: Putative pancreatic cancer-associated diabetogenic factor: $2030 \mathrm{MW}$ peptide. Pancreas 2002;24:8-14.

13 Libby G, Donnelly LA, Donnan PT, Alessi DR, Morris AD, Evans JM: New users of metformin are at low risk of incident cancer: a cohort study among people with type 2 diabetes. Diabetes Care 2009;32:1620-1625.

14 Mayerle J, Kalthoff H, Reszka R, Kamlage B, Peter E, Schniewind B, Maldonado SG, Pilarsky C, Heidecke $\mathrm{CD}$, Schatz P, Distler M: Metabolic biomarker signature to differentiate pancreatic ductal adenocarcinoma from chronic pancreatitis. Gut 2017, DOI: 10.1136/gutjnl-2016-312432.

15 Schultz NA, Dehlendorff C, Jensen BV, Bjerregaard JK, Nielsen KR, Bojesen SE, Calatayud D, Nielsen SE, Yilmaz M, Holländer NH, Andersen KK: MicroRNA biomarkers in whole blood for detection of pancreatic cancer. JAMA 2014;311:392-404.

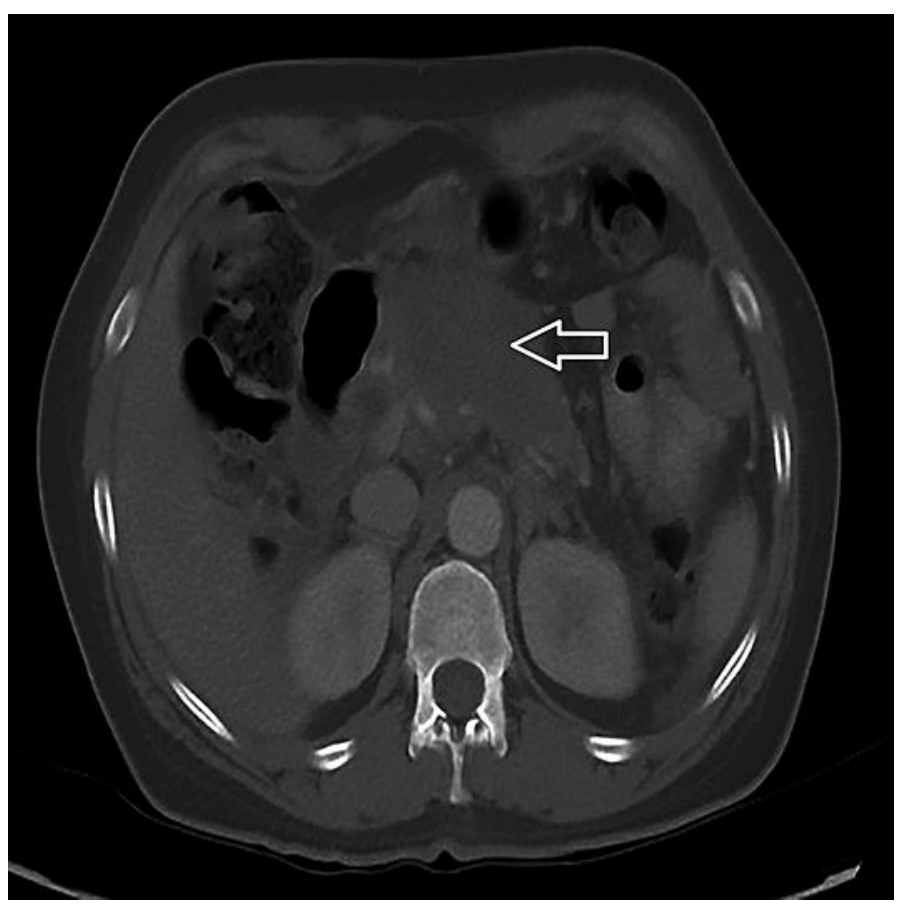

Fig. 1. CT of the abdomen and pelvis with contrast showed a 2.8-cm hypodense lesion (white arrow) in the hepatic dome with peripheral nodular enhancement, faint hypodense lesions in the lateral segment, and mild intrahepatic biliary dilatation. 


\section{Case Reports in Gastroenterology

\begin{tabular}{l|l}
\hline Case Rep Gastroenterol 2017;11:624-632 \\
\hline DOI: 10.1159/000481302 & $\begin{array}{l}\text { @ 2017 The Author(s). Published by S. Karger AG, Basel } \\
\text { www.karger.com/crg }\end{array}$ \\
\hline
\end{tabular} \\ Lin et al.: A Silent Asymptomatic Solid Pancreas Tumor in a Nonsmoking Athletic Female: Pancreatic Ductal Adenocarcinoma}

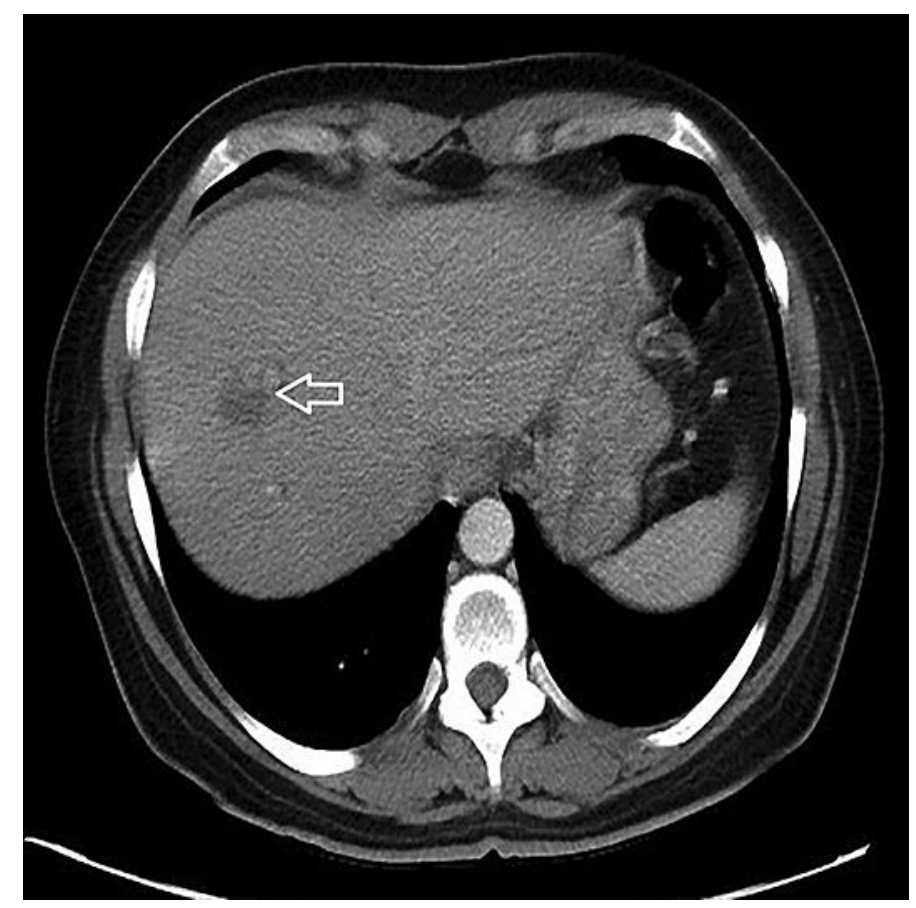

Fig. 2. There is a heterogeneous, irregular $8 \times 4.6 \mathrm{~cm}$ solid mass (white arrow) involving the pancreatic body with soft tissue extension to the celiac axis and superior mesenteric artery.

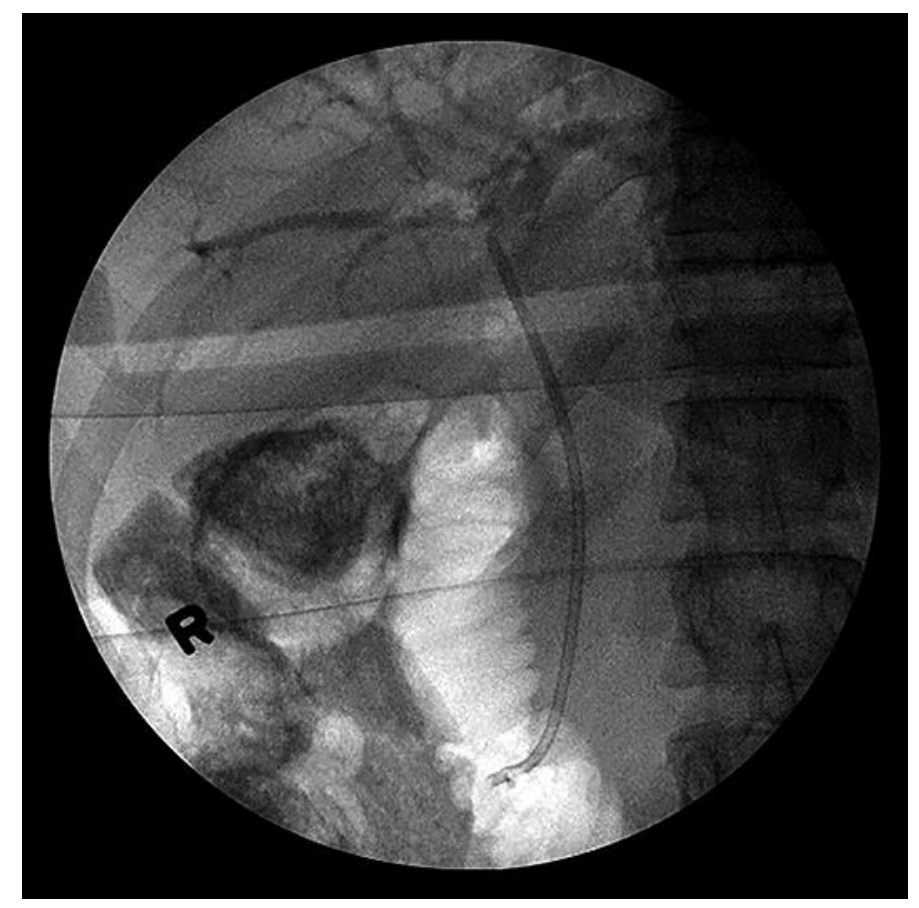

Fig. 3. Cholangiogram showed long segment $(3-4 \mathrm{~cm})$ distal to the CBD stricture with dilatation of mid and proximal CBD with dilated intrahepatics. 


\section{Case Reports in Gastroenterology Case Rep Gastroenterol 2017;11:624-632 \begin{tabular}{l|l} 
DOI: $10.1159 / 000481302$ & $\begin{array}{l}\text { @ } 2017 \text { The Author(s). Published by S. Karger AG, Basel } \\
\text { www.karger.com/crg }\end{array}$
\end{tabular} www.karger.com/crg \\ Lin et al.: A Silent Asymptomatic Solid Pancreas Tumor in a Nonsmoking Athletic Female: Pancreatic Ductal Adenocarcinoma}

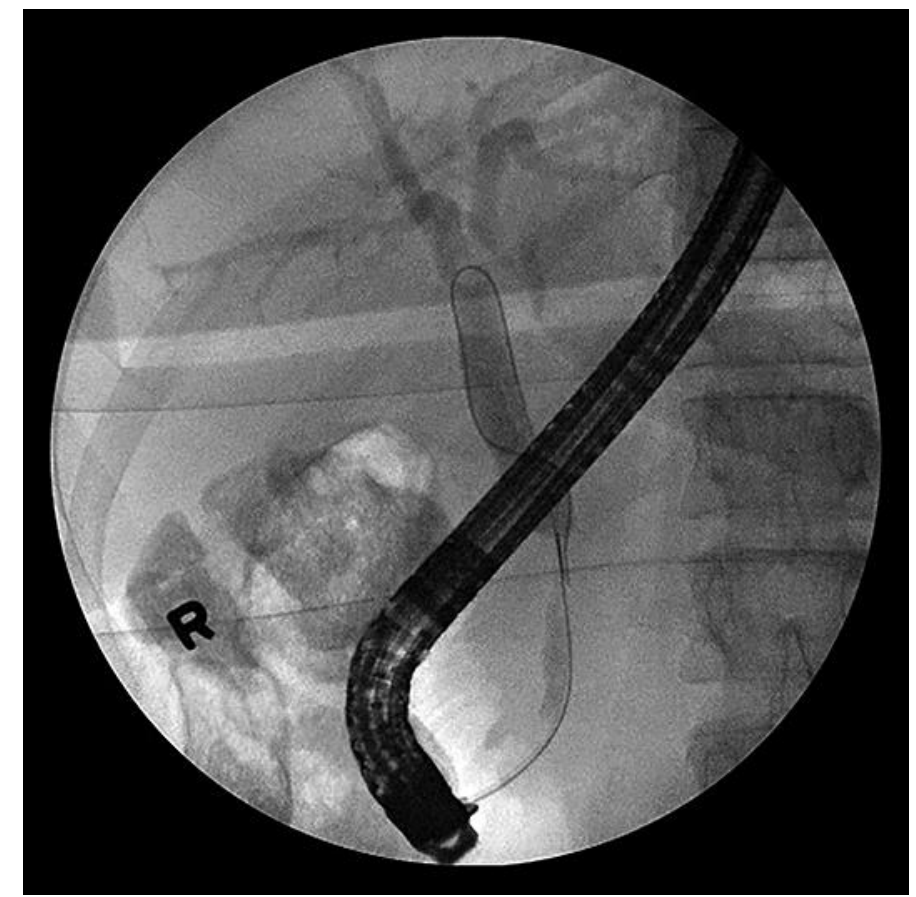

Fig. 4. ERCP showed CBD was cannulated at the ampulla using guidewire technique.

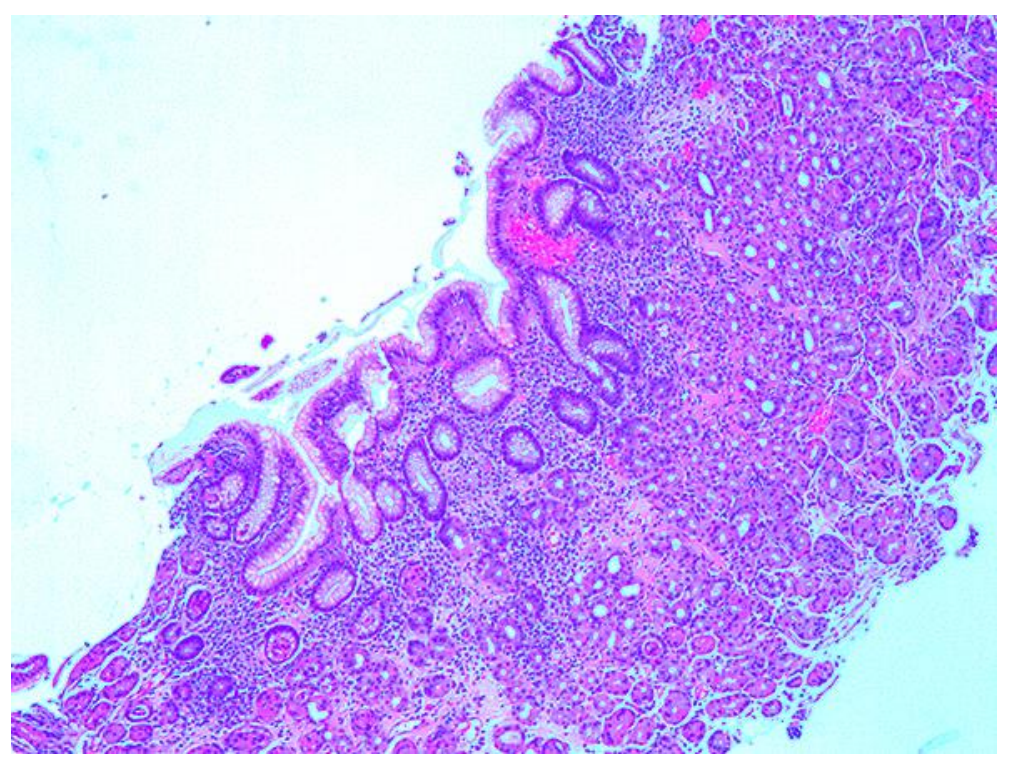

Fig. 5. Gastric biopsy $(\mathrm{HE}, \times 40)$ showed benign gastric mucosa with mild chronic gastritis. 
Lin et al:: A Silent Asymptomatic Solid Pancreas Tumor in a Nonsmoking Athletic Female: Pancreatic Ductal Adenocarcinoma

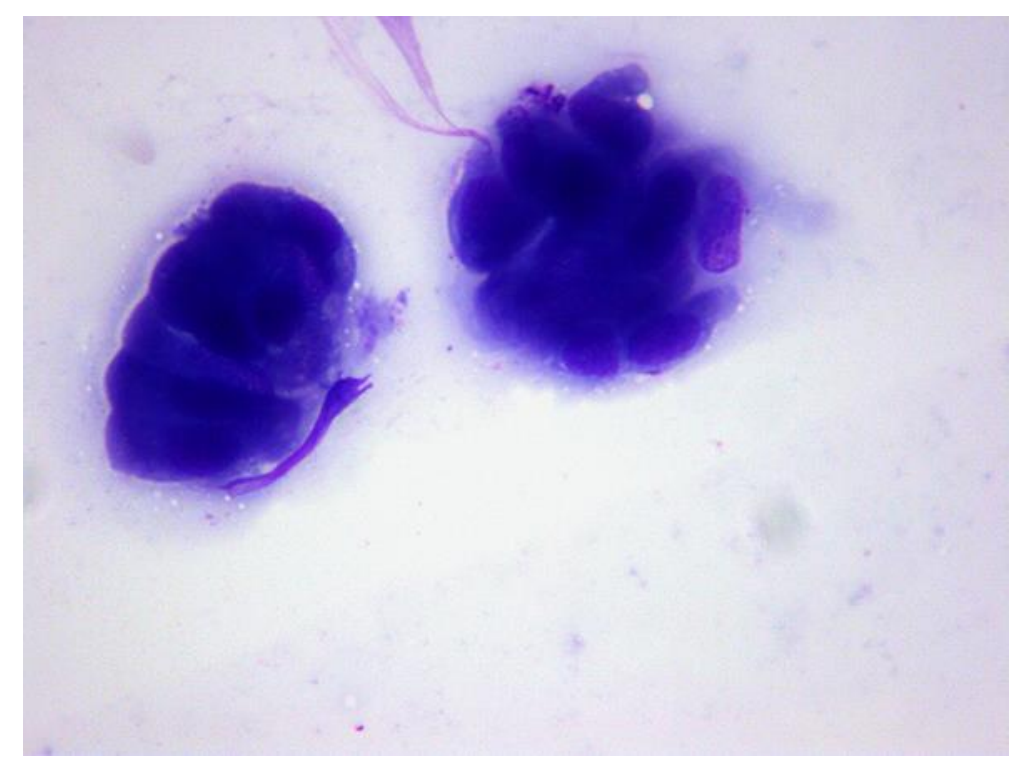

Fig. 6. EUS-guided fine needle aspiration biopsy $(\times 200)$ showed ductal epithelial cells with mild atypia. 